\title{
Patient and graft outcome of kidney transplantation during COVID-19 pandemic: a single center experience
}

\author{
Dianne Danielle Tan-Lim, Concesa Cabanayan-Casasola
}

Department of Adult Nephrology, National Kidney and Transplant Institute, Quezon City, Philippines

Background: Kidney transplantation (KT) rate is reduced globally due to the COVID pandemic. Although there have been reports on patients transplanted during the beginning of COVID-19, little is known about risk of doing KT in the Philippines during this pandemic, presentation and outcomes of COVID-19 infection among recipients immediately post-KT and how it will impact transplant outcomes. This study will aid clinicians in planning and management of KT during this pandemic. The objective of this study is to describe the patient and renal outcomes of Filipino patients who underwent KT in National Kidney and Transplant Institute during the period of COVID-19 pandemic.

Methods: A descriptive study of 147 patients aged $>18$ years old who underwent KT from June 1, 2020, to May 31, 2021. Peri-operative complications, graft and patient outcomes were noted during the study period.

Results: The median age was 43 years with $64 \%$ male and $59.2 \%$ with chronic glomerulonephritis as primary renal disease. It is notable that $12.9 \%$ were pre-emptive $\mathrm{KT}, 6.1 \%$ high immunologic risk and $49.7 \%$ given lymphocyte-depleting induction therapy. Around $98.6 \%$ were from living kidney donors with $1.4 \%$ deceased donor KT. Nine (6.12\%) had COVID pre-KT with median interval period from the last (+) rt-PCR COVID swab to KT of 150 days (range, 30-343 days). Infection rate post-KT was $14.2 \%$; majority was due to urinary tract infection. Around $2.7 \%$ had COVID infection post-KT with median interval period of 5 months post-KT (range, 1-8) with no mortality noted. The 6 -month acute rejection rate was $6 \%$ with graft and patient survival rate of $96.4 \%$ and $98.2 \%$, respectively.

Conclusions: COVID-19 pandemic has been challenging for our KT program, but our graft and patient outcomes are similar preCOVID. Further patient follow-up is needed to ascertain the outcome.

Corresponding author: Dianne Danielle Tan-Lim

E-mail: diannetanmd@gmail.com

(c) The Korean Society for Transplantation

This is an Open Access article distributed under the terms of the Creative Commons Attribution Non-Commercial License (http://creativecommons.org/licenses/by-nc/4.0/) which permits unrestricted non-commercial use, distribution, and reproduction in any medium, provided the original work is properly cited. 